\title{
Cutaneous Biology
}

\section{Changes in function and morphology of normal human skin: evaluation using optical coherence tomography}

\author{
J.WELZEL, C.REINHARDT, E.LANKENAU, * C.WINTER † AND H.H.WOLFF \\ Department of Dermatology, University of Lübeck, Ratzeburger Allee 160, D-23538 Lübeck, Germany \\ *Medical Laser Center Lübeck GmbH, Peter-Monnik-Weg 4, D-23562 Lübeck, Germany \\ †4optics AG, Roggenhorster Straße 29, D-23556 Lübeck, Germany
}

\begin{abstract}
Summary Background Optical coherence tomography (OCT) is a noninvasive morphological method for investigating human skin. It allows high-resolution in vivo imaging of inflammatory skin diseases and tumours. Because it is a newly developed method, systematic studies on standardization and on evaluation of factors influencing the representation of the skin have not yet been performed.

Objectives In this study, normal human skin was treated with various external stimuli which induce changes of function and morphology. Changes in stratum corneum thickness as well as changes induced by pigmentation, oedema and erythema were investigated using OCT.

Methods Healthy skin of human volunteers was treated with tape stripping, ultraviolet A irradiation, water, histamine, nicotinic acid and various ointments.

Results In the tape stripping experiment, the thickness of the horny layer was quantified and monitored. Pigmentation increased the light attenuation of the tissue, whereas hydration and erythema led to a slight decrease of scattering. Topical treatment of the skin gave a nonspecific increase of penetration depth of the light due to the lower reflectivity of the surface.

Conclusions There are various physiological conditions which influence optical properties of the skin. These parameters should be considered when performing standardized OCT studies.
\end{abstract}

Key words: morphology, optical coherence tomography, physiology, skin

Optical coherence tomography (OCT) has become an established method for in vivo imaging of tissue over the last 10 years. ${ }^{1}$ Early systematic studies of skin diseases demonstrated its value for monitoring inflammatory skin diseases and quantifying treatment effects. $^{2-7}$ Layers such as the epidermis and the dermis, adnexal structures and blood vessels can be well distinguished. The two-dimensional OCT images are comparable with ultrasound but have a higher resolution at the expense of penetration depth of the signal. The images are performed in real time without touching the surface of the skin. Using infrared radiation, the method is totally noninvasive. The two-dimensional images have a length of several millimetres and a penetration depth of about $1.5 \mathrm{~mm}$. The resolution of about $15 \mu \mathrm{m}$ allows detection of

Correspondence: Julia Welzel.

E-mail: welzel@medinf.mu-luebeck.de architectural details, but not visualization of single cells. Based on these specifications, the technique is of interest for noninvasive investigation of changes of the epidermis and the upper dermis.

Using ultrasound, it is known that various conditions such as gender, age, time of day, body posture and hormonal status influence the thickness and echodensity of the skin. ${ }^{8-10}$ Therefore, it is likely that there are several physiological conditions which influence skin optical properties. The aim of this study was to evaluate parameters which affect the representation of healthy skin in the OCT image.

The principle of OCT is based on interferometry. The light is coupled into optical fibres and divided into a reference beam with a known path length and a probe beam which is focused on to the skin. The light is scattered by the tissue and recombined again with the reference beam. Both beams interfere if they match to within the coherence length of the light source. Thus, 
the coherence length determines the axial resolution. Therefore, very short coherent, broad bandwidth light sources are used for OCT. By varying the path length of the reference beam, the interference modulation provides information on scattering in different depths of the probe. Lateral scanning of the signal results in a two-dimensional cross-sectional image of the tissue in which the lateral resolution depends on both the focusing and the step width. ${ }^{11}$

After illumination of the tissue, OCT as an optical method obtains image information from the backscattered or backreflected photons. The scattering depends on the size and shape of the particles inside the tissue. Light absorption is influenced by both the wavelength and the composition of the tissue. Melanin, haemoglobin and water have a wavelength-dependent absorption coefficient. For receiving a high penetration depth of the signal, radiation sources with a wavelength in the near infrared are used for OCT where absorption is relatively low. Reflection occurs if the light enters into a medium with a different refractive index. Therefore, a major part of the light is reflected from the surface when the medium changes from air to skin which has a refractive index of about $1 \cdot 33$, similar to water. ${ }^{12}$ Index matching using water as a coupling medium reduces the reflection.

On the assumption that melanin, haemoglobin, water and the distribution of particles influence the optical properties of skin, it is likely that changes of these parameters are visible and detectable in the OCT image. In this study, the effect of pigmentation, reddening, oedema and thickness of the stratum corneum was evaluated. Additionally, the influence of



various topical treatments on the reflectivity of the surface and the penetration depth of the signal was investigated.

\section{Subjects and methods}

Optical coherence tomography system

A prototype of the OCT system Sirius (4optics, Lübeck, Germany) with an axial and lateral resolution of about $15 \mu \mathrm{m}$ was used for this study. The fibre-optic light source has a wavelength of $1300 \mathrm{~nm}$. The two-dimensional images have a lateral dimension of 4-6 $\mathrm{mm}$ and a detection depth of about $1 \mathrm{~mm}$. A compact handpiece allows the study of any skin region. In an area of interest, an average a-scan, which represents the depth resolved signal intensities in the probe, can be calculated. On the curve, distances between peaks, signal intensities and the signal attenuation coefficient $(\mu)$ as a function of the slope of the curve can be calculated at different depths (Fig. 1). The study and the OCT measurements were performed with the consent of the local ethics committee.

\section{Other noninvasive methods}

Measurements of the transepidermal water loss (TEWL) were performed using the Tewameter TM 210 (Courage \& Khazaka, Cologne, Germany). For investigations of skin colour, the Chromameter CR 200 (Minolta, Ahrensburg, Germany) and the Mexameter MX 16 (Courage \& Khazaka) were used. Bioengineering methods were used regarding the guidelines for

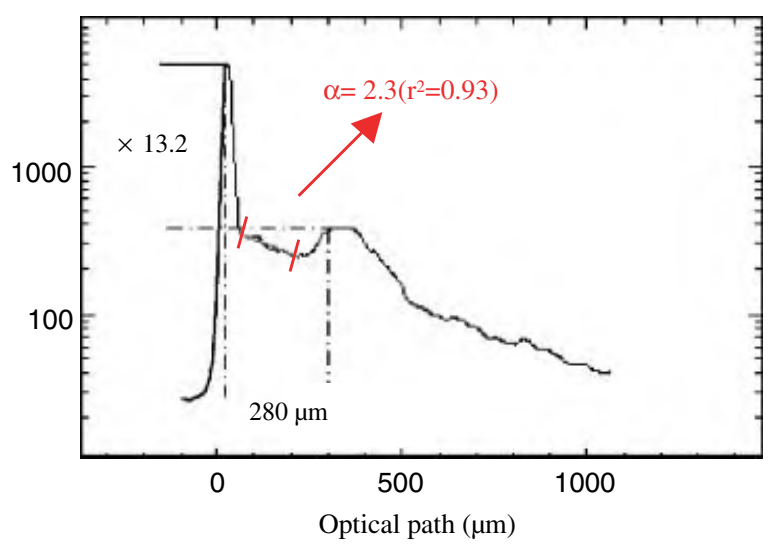

Figure 1. Two-dimensional optical coherence tomography image of the fingertip (left) and average a-scan (right) with calculation of the height of peaks, distances between peaks and the slope of the curve representing the light attenuation coefficient ( $\mu$ ). The first intensity peak representing the surface is $13 \cdot 2$ times higher than the second peak at the border between the stratum corneum and living epidermis. The thickness of the stratum corneum is $280 \mu \mathrm{m}$. The attenuation coefficient is calculated inside the stratum corneum (red line) and is the slope of the curve $\left(2 \cdot 3 \mathrm{~mm}^{-1}\right)$. 
standardization, i.e. at a constant air temperature and humidity after acclimatizing for $15 \mathrm{~min}$.

\section{Subjects}

Prior to the study, 10 consecutive OCT images of the same area at the forearm of a healthy male volunteer (age 27 years) were taken over a period of $6 \mathrm{~h}$. These measurements were repeated after 3 days to determine the variation of the values without any treatment. A template was used to find the same region again.

The treatments and measurements were performed on healthy skin of 34 human volunteers ( 28 women, six men; age range 24-57 years) after informed consent. Tape stripping and pigmentation were investigated in eight subjects. Oedema and erythema were induced in 15 subjects and the skin of another 15 subjects was treated with different ointments and creams.

\section{Treatments}

Tape stripping. Consecutive tape strippings were performed at the palm (thenar region) and at the inner forearm using the adhesive tape Tesa ${ }^{\circledR}$ (Beiersdorf, Hamburg, Germany). The treatment was repeated until the TEWL increased up to $30 \mathrm{~g} \mathrm{~m}^{-2} \mathrm{~h}^{-1}$. Approximately 30-40 consecutive tape strips were necessary to reach this endpoint. OCT images were collected before and after tape stripping.

Pigmentation. On gluteal skin, a single ultraviolet (UV) A irradiation of $120 \mathrm{~J} \mathrm{~cm}^{-2}$ was applied. OCT and skin colour measurements were performed before treatment, after $1 \mathrm{~h}$ and after $24 \mathrm{~h}$ following treatment.

Oedema and erythema. At the inner forearm, the dermal water content was increased by intracutaneous application of histamine. At an adjacent area, an ointment containing nicotinic acid (Finalgon Extra Stark ${ }^{\circledR}$ ) was applied to produce erythema. OCT images of both test sites and of the adjacent untreated skin were taken before treatment and after $20 \mathrm{~min}$.

Topical treatments. Different treatments were performed at each fingertip of one hand. At the distal palmar part, $0.01 \mathrm{~mL}$ of glycerol, paraffin oil, petrolatum, $10 \%$ urea in petrolatum and ultrasonic gel (containing 15\% glycerol in water) were applied. OCT measurements were performed before and directly after treatment. The images were compared with the respective, untreated fingertips of the other hand.

\section{Statistical analysis}

The Wilcoxon signed rank test for matched pairs was used to compare the values of the noninvasive methods before and after treatment. The same test was performed for evaluating differences between treated and untreated skin within a single subject. Significance was assumed at $P \leq 0 \cdot 05$. The different methods were compared by calculating the Spearman rank correlation coefficient with significance of $r_{\mathrm{s}}>+0.5$ or $<-0.5$, respectively.

\section{Results}

It is vital to know the typical appearance of healthy skin at different anatomical regions when interpreting OCT. Images of palmoplantar skin display a wavy surface due to the dermatoglyphics. The first layer is thick, homogeneous, with only a few weak signals and is sharply demarcated from the next layer (Fig. 2A). It corresponds to the stratum corneum. The living epidermis and the upper dermis are not well defined. In forearm skin, the stratum corneum is not thick enough to be visible at this resolution. The superficial layer is the epidermis, which shows a well-defined border to the upper dermis. The dermis is more signal intense with some signal-poor cavities corresponding to blood vessels (Fig. 3).
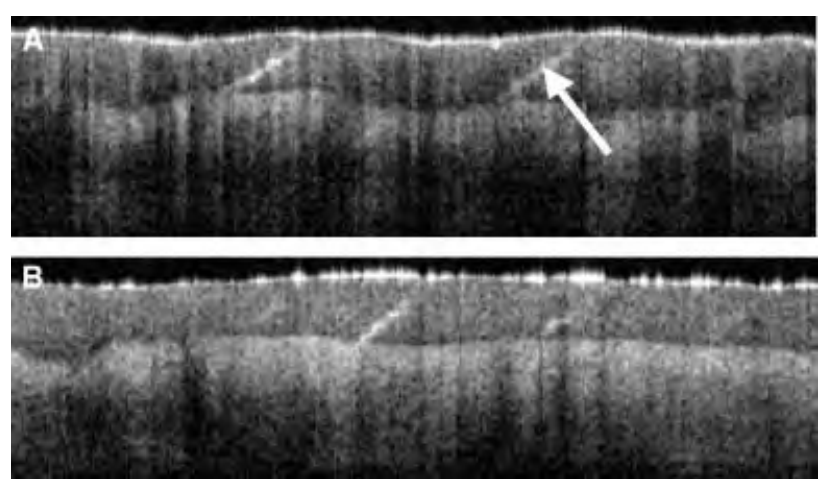

Figure 2. Optical coherence tomography image of healthy skin of a fingertip. The first wavy layer is the thick stratum corneum. Some spiral sweat gland ducts inside are visible (arrow). The image above (A) shows untreated skin. Below (B), the effect of glycerol directly after application at the surface of the same fingertip is demonstrated. The entrance signal is irregular with some interruptions. Deeper parts of the image are slightly more signal intense. Image area $4 \times 1 \cdot 1 \mathrm{~mm}$. 


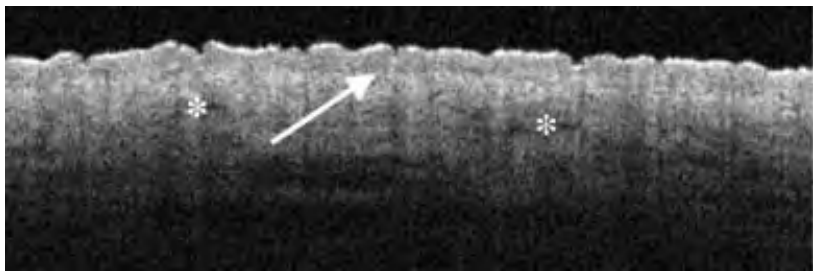

Figure 3. Healthy forearm skin. The stratum corneum is not detectable. The first layer is the epidermis. The border to the dermis is marked by an arrow, whereas blood vessels are seen as signal-free cavities indicated by ${ }^{*}$. Image area $4 \times 1.3 \mathrm{~mm}$.
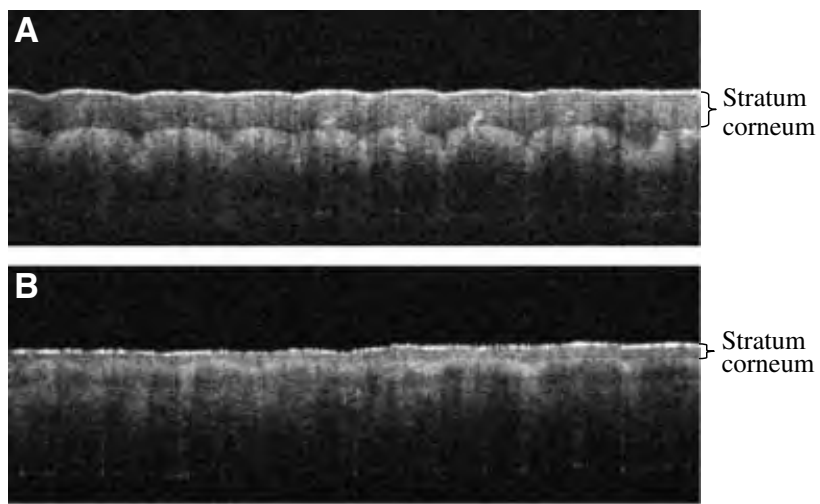

Figure 4. Optical coherence tomography image of the palm before (A) and after consecutive tape stripping (B). The stratum corneum is thinner but is still visible. Image area $4 \times 1 \cdot 1 \mathrm{~mm}$.

The values of repeated distance and light attenuation measurements of the same area showed a variation of about $10 \%$ without treatment. After tape stripping, the stratum corneum at the palm showed a stepwise decrease in thickness from $203 \mu \mathrm{m}$ to $170 \mu \mathrm{m}$ (Fig. 4). This was quantified by measuring the distance between the entrance signal of the surface and the intensity peak representing the border between the stratum corneum and the living epidermis. Although the horny layer was not visible at the forearm, the distance between the entrance peak and the upper dermis also decreased at this location. The mean value before tape stripping was $132 \mu \mathrm{m}$ and this was reduced to $115 \mu \mathrm{m}$ after stripping. The light attenuation coefficient $(\mu)$ of the dermis was not significantly affected by the treatment.

Experimental UVA irradiation gave rise to a significant increase of the $\mathrm{a}^{*}$-value of the colour measurement after $1 \mathrm{~h}$, representing the immediate erythema. The melanin index increased slightly after $24 \mathrm{~h}$ (not significant). In the OCT images, the thickness of the epidermis and the light attenuation inside the epider-
Table 1. Light attenuation coefficient $(\mu)\left[\mathrm{cm}^{-1}\right]$ of the upper dermis before and $20 \mathrm{~min}$ after treatment with histamine or nicotinic acid. Mean \pm SD, $n=15$

\begin{tabular}{lccc}
\hline & Histamine & Nicotinic acid & Untreated control \\
\hline Before treatment & $19 \cdot 8 \pm 3 \cdot 1$ & $19 \cdot 4 \pm 3 \cdot 0$ & $21 \cdot 1 \pm 2 \cdot 0$ \\
After 20 min & $19 \cdot 0 \pm 3 \cdot 2$ & $18 \cdot 8 \pm 3 \cdot 8$ & $21 \cdot 8 \pm 3 \cdot 6$ \\
\hline
\end{tabular}

Table 2. Influence of various treatments of the skin surface on the intensity of the entrance signal (relation between the peak and the noise level, without dimension) and on the light attenuation coefficient $(\mu)\left[\mathrm{cm}^{-1}\right]$ of the stratum corneum. Mean \pm SD, $n=15$. Values in parenthesis represent untreated control

\begin{tabular}{lll}
\hline Treatment & $\begin{array}{c}\text { Entrance peak/ } \\
\text { background noise }\end{array}$ & $\begin{array}{c}\mu \text { stratum } \\
\text { corneum }\left[\mathrm{cm}^{-1}\right]\end{array}$ \\
\hline Ultrasonic gel, thumb & $60 \pm 22$ & $21 \pm 10$ \\
(opposite untreated control) & $(108 \pm 56)^{* *}$ & $(26 \pm 8) \mathrm{NS}$ \\
Paraffin oil, index finger & $101 \pm 67$ & $17 \pm 14$ \\
(opposite untreated control) & $(143 \pm 51) \mathrm{NS}$ & $(27 \pm 9)^{*}$ \\
10\% urea, middle finger & $104 \pm 52$ & $20 \pm 14$ \\
(opposite untreated control) & $(131 \pm 42)^{*}$ & $(24 \pm 10) \mathrm{NS}$ \\
Glycerol, ring finger & $106 \pm 60$ & $19 \pm 18$ \\
(opposite untreated control) & $(130 \pm 51) \mathrm{NS}$ & $(24 \pm 14) \mathrm{NS}$ \\
Petrolatum, little finger & $82 \pm 52$ & $14 \pm 18$ \\
(opposite untreated control) & $(128 \pm 56)^{*}$ & $(24 \pm 13) \mathrm{NS}$ \\
\hline
\end{tabular}

NS, not significant, ${ }^{*} P \leq 0 \cdot 05,{ }^{* *} P \leq 0 \cdot 01$.

mis were not changed, whereas after $24 \mathrm{~h}$ the signal attenuation coefficient of the upper dermis increased significantly from $39 \pm 14 \mathrm{~cm}^{-1}$ to $47 \pm 23 \mathrm{~cm}^{-1}$. The $\mathrm{a}^{*}$-value for the colour red and the melanin index showed no significant correlation to the light attenuation.

After induction of dermal changes due to erythema and oedema, the light attenuation was slightly decreased. This was not significant compared with the values before treatment, but reached significance compared with the light attenuation of the untreated adjacent skin (Table 1 ).

Different topical treatments of the fingertips were all followed by a decrease of the reflectivity of the skin surface, represented by a lower entrance peak in the average a-scan. The penetration depth of the light signal increased, which was due to a lower light attenuation. The comparison between the different products showed gradual differences. The influence on light attenuation was greatest for paraffin oil (Table 2, Figs 2B and 5).

\section{Discussion}

Various conditions were found to have an influence on optical properties of the skin. The tape stripping study 


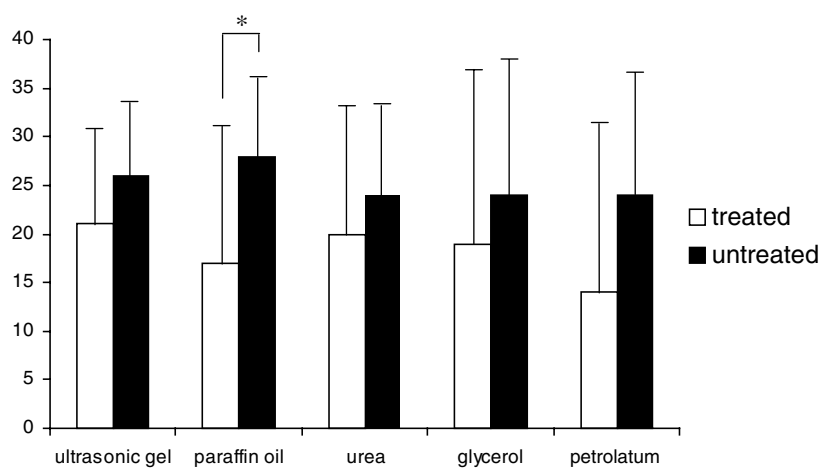

Figure 5. Influence of various treatments of the skin surface on the light attenuation coefficient $(\mu)\left[\mathrm{cm}^{-1}\right]$ inside the stratum corneum. Mean \pm SD, $n=15 .{ }^{*} P \leq 0 \cdot 05$.

demonstrated that the thickness of the stratum corneum could be quantified during consecutive strippings. This might be of interest for further investigations regarding the effect of the skin barrier on healing processes, because OCT allows for noninvasive morphological monitoring rather than employing indirect methods such as TEWL measurements. Even at regions with a thin horny layer such as the forearm, tape stripping led to a detectable decrease of thickness of the epidermis, although this effect was not visible by the naked eye in the OCT images due to low resolution of the OCT system. The light attenuation coefficient of deeper skin layers was not affected by the removal of the stratum corneum. This indicates that different skin structures do not influence each others' optical properties.

Experimentally induced pigmentation of the epidermis as well as the fluid content of the dermis did not greatly affect the light attenuation coefficient $(\mu)$, although some statistically significant changes were observed. The light attenuation is influenced by both scattering and absorption. At a wavelength of $1300 \mathrm{~nm}$, the effect of scattering is higher than the degree of absorption. ${ }^{13}$ Therefore, changes in the distribution, size, density and orientation of particles are of a higher importance than the content of melanin, haemoglobin and water. Irradiation induces an increase of the melanin concentration. This is followed by a higher light scattering in the epidermis and a loss of penetration depth of the light into deeper regions of the skin. This might explain the increase of the light attenuation coefficient in the upper dermis. Erythema is due to a dilation of blood vessels as a morphological correlate, accompanied by an increase of haemoglobin. In the same way, oedema changes both the orientation and density of collagen fibres as well as dermal water content. The morphological changes caused by increased hydration and perfusion of the dermis may explain why lower light attenuation was observed (caused by a decrease of the scattering by the collagen bundles). It is known from ultrasound studies that dermal oedema reduces the echodensity of the dermis due to changes in collagen orientation. ${ }^{8-10}$ The influence of water- and haemoglobin-dependent higher absorption is not strong enough to weaken this effect. The water absorption coefficient is remarkably increased only at higher wavelengths above $1500 \mathrm{~nm}$.

Treatment of the skin surface lowers the intensity of the entrance peak and increases the penetration depth of the light. This effect was nonspecific and could be observed in all treatment groups irrespective of the chemical composition (water vs. alcohol vs. fat). Decreased light attenuation was also not caused by influencing the water-binding capacity or water content of the stratum corneum, as it was observed directly after treatment and did not change when using moisturizers (glycerol or urea). The increase of penetration depth is the result of decreased skin surface reflectivity due to the treatment. All products influenced the opacity of the horny layer, which became more transparent. This effect is comparable with the increase in transparency of wet or greasy paper and is also the reason why immersion oil is used for conventional or epiluminescence microscopy to improve image quality.

In summary, we found that stratum corneum thickness does not influence the optical properties of deeper layers. Tape stripping studies could be monitored using OCT. Pigmentation decreased the signal intensity in the dermis slightly, due to a higher scattering by melanin. Erythema and oedema decreased light attenuation, which is likely to be caused by lower scattering in the dermis. These influences should be considered when performing standardized OCT studies. Moreover, OCT allows a quantification of these effects and may therefore be an interesting additional bioengineering method for monitoring inflammatory skin diseases and therapeutic effects. Topical treatment of the skin surface improves the image quality. Paraffin oil, glycerol or ultrasonic gel may be used for routine OCT measurements, but it is not imperative to use any substance for refractive index matching or coupling the applicator on the skin. When performing OCT studies on therapeutic effects, no further products should be applied in order to avoid artifacts. 


\section{Acknowledgments}

This work was supported by the Deutsche Forschungsgemeinschaft (DFG We 2117/4-1).

\section{References}

1 Huang D, Swanson EA, Lin CP et al. Optical coherence tomography. Science 1991; 254: 1178-81.

2 Welzel J, Lankenau E, Birngruber R, Engelhardt R. Optical coherence tomography of the human skin. J Am Acad Dermatol 1997; 37: 958-63.

3 Hoffmann K, Happe M, Fricke B et al. Optical Coherence Tomography (OCT) in der Dermatologie. In: Dermatologie - Leitlinien und Qualitätssicherung für Diagnostik und Therapie (Garbe C, Rassner G, eds). Berlin: Springer-Verlag, 1998: 3-8.

4 Pagnoni A, Knüttel A, Welker P et al. Optical coherence tomography in dermatology. Skin Res Technol 1999; 5: 83-7.

5 Gladkova ND, Petrova GA, Nikulin NK et al. In vivo optical coherence tomography imaging of human skin: norm and pathology. Skin Res Technol 2000; 6: 6-16.

6 Welzel J. Optical coherence tomography in dermatology: a review. Skin Res Technol 2001; 7: 1-9.
7 Welzel J, Noack J, Lankenau E, Engelhardt R. Optical coherence tomography in dermatology. In: Handbook of Optical Coherence Tomography (Bouma BE, Tearney GJ, eds). New York: Marcel Dekker, 2001; 539-61.

8 Gniadecka M, Serup J, Söndergaard J. Age-related diurnal changes of dermal oedema: evaluation by high-frequency ultrasound. Br J Dermatol 1994; 131: 849-55.

9 Eisenbeiß C, Welzel J, Schmeller W. The influence of female sex hormones on skin thickness: evaluation using $20 \mathrm{MHz}$ sonography. Br J Dermatol 1998; 139: 462-7.

10 Eisenbeiß C, Welzel J, Eichler W, Klotz K. Influence of body water distribution on skin thickness: measurements using highfrequency ultrasound. Br J Dermatol 2001; 144: 947-51.

11 Bouma BE, Tearney GJ (eds). Handbook of Optical Coherence Tomography. New York: Marcel Dekker, 2001.

12 Knüttel A, Böhlau-Godau M. Spatially confined and temporally resolved refractive index and scattering evaluation in human skin performed with optical coherence tomography. J Biomed Opt 2000; 5: 83-92.

13 Troy TL, Thennadil SN. Optical properties of human skin in the near infrared wavelength range of 1000-2200 nm. J Biomed Opt 2001; 6: 167-76. 